\title{
- Reduced uncertainty in temperature projections using . carbon cycle and climate observations
}

3 Roger Bodman ${ }^{1,2}$, Peter Rayner ${ }^{1} \&$ David Karoly ${ }^{1}$

${ }_{4}{ }^{1}$ The University of Melbourne, Melbourne, Australia.

${ }_{5}^{2}$ Victoria University, Melbourne, Australia.

6 The future behaviour of the carbon cycle is a major contributor to uncertainty in temper7 ature projections for the 21st century. Using a simplified climate model, we show that, for 8 a given emission scenario, it is the second most important contributor to this uncertainty 9 after climate sensitivity, followed by aerosol impacts. Historical measurements of carbon dioxide concentrations have been used along with global temperature observations to

11 help reduce this uncertainty. This results in an increased probability of exceeding a $2^{\circ} \mathrm{C}$ 12 global-mean temperature increase by 2100 while reducing the probability of surpassing a $6^{\circ} \mathrm{C}$ threshold for non-mitigation scenarios such as the SRES A1B and A1FI scenarios ${ }^{1}$, 


\section{Introduction}

The last decade has seen a great deal of research into quantifying the uncertainty of climate projections as a guide to sensible adaptation and mitigation activity. The two most common techniques have been multi-model ensembles (such as the CMIP3, Third Coupled Model Intercomparison Project ${ }^{2}$ and C4MIP, Coupled Carbon Cycle Climate Model Intercomparison Project ${ }^{3}$ ) and perturbed physics experiments ${ }^{4}$. These experiments have allowed us to isolate those factors responsible for the spread of model ensembles. They have identified factors contributing to the overall climate sensitivity and uncertainty in the radiative response to aerosol changes, as well as the response of the carbon cycle, including some assessment of the feedbacks between climate change and carbon fluxes.

More recently, observations have been used to provide direct constraints on model behaviour ${ }^{5,6}$. Computational complexity has largely precluded the use of full Earth system models (ESMs) and restricted the facets of model behaviour that can be constrained. Full ESMs generally explore fewer model parameters or use statistical emulation to expand their sample size. Most critically, the carbon cycle behaviour remains largely untreated (with a rare exception ${ }^{7}$ ), despite the sensitivity of global-mean temperature change projections to carbon cycle processes ${ }^{8}$. Instead, typically, simple models have been calibrated against the coupled carbon cycle/climate models of Friedlingstein et al. ${ }^{3}$ for which only limited formal calibration is possible and which do not address parametric uncertainty.

Here we apply historical observations to address the scientific uncertainty of the climate system. Our general method has been to identify those model parameters most responsible for 
uncertainty in global-mean temperature changes, and then to constrain them with historical observations. The model used was MAGICC version 6.3 (Model for the Assessment of Greenhouse Gas Induced Climate Change), a reduced complexity Earth System Model ${ }^{9}$, whose climate system parameters have previously been estimated using historical observations ${ }^{6}$. More recently, the climate system and carbon cycle parameters have been calibrated against both CMIP3 and C4MIP models9.

MAGICC has been involved in recent studies of temperature change projections using SRES and RCP scenarios ${ }^{10,11}$. However, as noted in Rogelj et al. ${ }^{11}$, calibration with just the C4MIP models neglects structural and methodological uncertainties and is therefore unlikely to represent the range of carbon cycle uncertainties. Here, for the first time, observations have been used to directly constrain the model's carbon cycle parameters. We then generate probability distributions for global-mean temperature change that reflect uncertainties for the combined climate system and carbon cycle, taking into account a comprehensive range of model and observational uncertainties.

\section{Contribution to uncertainty}

We see that, before using observations to improve prediction, the climate sensitivity, carbon cycle response and aerosol impacts are the three leading contributors to uncertainty (in that order). This was determined by a linear analysis of the uncertainty contributions to the simulated temperature change in 2100 from the model's primary parameters. Prior probability distributions for the model's parameters were estimated based on previous calibration exercises ${ }^{6,9}$ and earlier studies ${ }^{12}$, although larger uncertainties were used for the carbon cycle parameters (see 
Methods and Supplementary Information for further details).

The uncertainty contributions for the A1FI emission scenario show that about $63 \%$ of the uncertainty stems from the climate system parameters, with over $90 \%$ of this due to the climate sensitivity parameter (Table 1), while just three additional parameters account for much of the remainder (ocean vertical diffusivity, land ocean warming ratio and the land ocean heat exchange coefficient). The carbon cycle accounts for around $30 \%$ of the uncertainty in temperature change in 2100 , most of which is captured by six parameters: two temperature feedback parameters for respiration and net primary productivity (NPP), plus the $\mathrm{CO}_{2}$ fertilisation factor, the fraction of NPP to plant and plant to detritus, plus an ocean carbon cycle impulse response scaling factor. A single parameter was used for the aerosol forcing uncertainty, the fossil fuel derived sulfate aerosol forcing in 2005, with the indirect or cloud albedo effect scaled from the total direct effect. This set of eleven parameters accounts for $96 \%$ of the model's parametric uncertainty.

\section{[Table 1]}

\section{Constraining with observations}

The Monte Carlo Metropolis-Hastings algorithm (MCMH) was applied to constrain the eleven model parameters identified above using 20th-century observations. These included globalmean temperature change, land minus ocean and hemispheric temperature differences ${ }^{13}$, time series for ocean heat content changes ${ }^{14}$, and a 1960-2008 ocean vertical temperature change profile ${ }^{15}$. Observations for the carbon cycle employed atmospheric $\mathrm{CO}_{2}$ concentrations from 
Mauna Loa ${ }^{16}$.

Details of the resulting posterior distributions for the parameters are provided in Supplementary Table S1 and Figure S1. Note that individual distributions are not independent, so that, for example, the distribution for climate sensitivity is not a stand-alone result since the observational constraints introduce sizeable correlations between some of the parameters (parameter correlations are included in Supplementary Table S2). For example, a strong aerosol effect (which reduces temperature changes) requires a higher climate sensitivity to match the observed temperature changes, as found in other studies ${ }^{17}$.

We repeated the linear uncertainty analysis with the posterior covariance to determine which parameters and combinations are most responsible for the improved knowledge of projected temperature change (Supplementary Figure S3). About $60 \%$ of the reduction in temperature standard deviation comes from uncertainty reduction in individual parameters with the other $40 \%$ arising from parameter covariance. The largest single contribution comes from the climate sensitivity. There is also a large contribution from combinations of carbon cycle parameters even though the individual parameters are not well-constrained. Studies that do not consider these covariances are likely to overestimate uncertainty in temperature change projections.

To assess the benefit of constraining the model's parameters using the carbon cycle observations, we compared three ensembles of predicted temperature change for 2100 under the SRES A1FI scenario. Ensembles were generated by constraining model parameters with different combinations of constraints. The 'Combined' ensemble used both climate and carbon cycle 
The results (Table 2) reveal the benefit of including carbon cycle observations in the $\mathrm{MCMH}$ algorithm, where the effect is to limit the spread in the right-hand side of the probability distribution, reducing the likelihood of very high $\mathrm{CO}_{2}$ concentration and temperature changes by 2100 .

[Table 2]

\section{Probabilistic temperature projections}

The observationally constrained posterior parameter distribution was applied to the SRES A1FI, $\mathrm{A} 1 \mathrm{~B}$ and $\mathrm{A} 2$ emission scenarios. Details for temperature change and $\mathrm{CO}_{2}$ concentrations in 2100 are provided for the prior and posterior distributions, with and without the carbon cycle temperature feedbacks (Table 3), while the global-mean temperature change results are illustrated in Figure 1.

[Table 3]

[Figure 1]

The median, mode and likely ranges for temperature change in 2090-2099 were com- 
pared to the IPCC AR4 best estimate and likely range results for these three scenarios and illustrated by the uncertainty bars in Figure 1 (numerical details are set out in Supplementary Table S4). The MAGICC mode temperature changes at 2090-2099 are almost the same as the AR4 best estimates for the A1B and A1FI scenarios, while the likely ranges are reduced at both extremes. This increases the probability of exceeding a $2{ }^{\circ} \mathrm{C}$ global-mean temperature increase by 2100 and reduces the probability of surpassing a $6^{\circ} \mathrm{C}$ threshold. The differences arise from the observational constraints imposed by the MCMH calibration process, with the interaction of the individual asymmetric parameter distributions, the interplay of the uncertainties from both the carbon cycle and aerosol radiative forcing, and the amount of sulfur dioxide emissions in the individual scenarios. The central estimate and upper bound for the A2 scenario are noticeably cooler than the AR4 estimates as a result of the higher aerosol cooling.

Projections based on the prior and posterior distributions with and without the carbon cycle temperature feedbacks (Table 3) show how the uncertainty ranges are reduced, although the central estimates reveal little change. The impact of the carbon cycle temperature feedbacks are also evident, with increases in $\mathrm{CO}_{2}$ concentrations demonstrating a net positive feedback effect. The likely ranges are also wider with the temperature feedbacks on, indicating how emissions driven temperature change projections are affected by both the carbon cycle feedbacks and the uncertainties in those feedbacks. Concentration driven projections will underestimate the amount of temperature change unless this issue is allowed for. 


\section{Probability of exceeding $2^{\circ} \mathrm{C}$}

The posterior parameter distributions also allow an assessment of the probability of exceeding selected temperature targets. For example, for the $2^{\circ} \mathrm{C}$ target associated with dangerous climate change $^{18,19}$, Figure 2 plots the three SRES scenarios for (a) global-mean temperature change and (b) land only surface temperature changes, relative to pre-industrial (rather than relative to 1980-1999 as in the previous figures and tables).

[Figure 2]

The A1B scenario shows a slightly increased probability of exceeding $2^{\circ} \mathrm{C}$ as compared to A1FI at the start of the 21 st-century because of higher initial $\mathrm{CO}_{2}$ emissions. The A1FI scenario has a greater than $50 \%$ chance of exceeding $2^{\circ} \mathrm{C}$ by around 2045 , although over the land surface this $50 \%$ is exceeded a decade earlier, around 2035. By the end of this century, the probability of exceeding $2^{\circ} \mathrm{C}$ global-mean temperature change is more than $95 \%$, whilst the probability over land is close to $100 \%$ for all three scenarios.

Limitations in this work stem from inadequacies in the model and our estimation process. MAGICC is a simplified ESM which lacks certain processes capable of increasing the uncertainty (e.g., water and nutrient cycles and liberation of carbon in permafrost). It also removes the state dependence of other processes such as ocean diffusivity or surface albedo. Finally, a single model cannot explicitly consider structural uncertainty ${ }^{20}$ although it can be included in the observational uncertainty ${ }^{21}$. 
The treatment of prior PDFs in Bayesian calibration always requires care ${ }^{20,22}$. The main

results of this paper are, however, based on reasonable assumptions or are insensitive to the choice of prior. The extra information available from carbon cycle observations does depend on the prior since, if there is little prior uncertainty, there is little to add. We agree with Rogelj et al. ${ }^{11}$ that C4MIP ranges underestimate total uncertainty and expanded them accordingly. The impact of uncertainty covariance on temperature projections depends only weakly on the magnitude of the posterior uncertainty itself. The specification of data uncertainty is also beset with difficulty. Here we have used only decadal averaged data consistent with the capabilities of MAGICC and have included observation error correlations to deal with potential persistent errors.

The experiments presented here were based on selected SRES emission scenarios for the purposes of comparison to previous studies. However, emission scenarios need to be reassessed to allow for revised expectations in global economic growth, energy intensity, per capita consumption and demography. In addition, it would be useful to provide probabilistic temperature change projections using the RCPs (Representative Concentration Pathways), perhaps following Rogelj et al. ${ }^{11}$.

\section{Methods}

Sensitivity analysis. MAGICC was run with a nominally standard set of initial parameter values (10 climate, one combined aerosol and 18 carbon cycle parameters) and A1FI emissions to establish a reference temperature change, then re-run for each parameter in turn, with the parameter value changed by $1 \%$ of its standard deviation. The difference in the year 2100 
temperature change results then provides an uncertainty measurement of the model outputs for each of the input parameters (the Jacobian of the model $\mathbf{J})^{23}$. The variance of each parameter provides the content for the covariance matrix $\mathbf{C}(v)$. To first order, the uncertainty of the temperature projection $\mathbf{U}$ is given by:

$$
\mathbf{U}=\mathbf{J C}(v) \mathbf{J}^{\mathbf{T}}
$$

where the superscript represents the transpose of the matrix $\mathbf{J}$.

The results, that is, the relative contributions to uncertainty from the primary model parameters for the A1FI emission scenario, are provided in Table 1 (the sensitivity of these results to the emissions pathway, initial parameter values and second-order effects is discussed in the Supplementary Information).

Monte Carlo Metropolis-Hastings algorithm. Our implementation of the Monte Carlo MetropolisHastings (MCMH) algorithm compares model results from each iteration to a combined set of historical climate and carbon cycle observations, applying a decision rule to accept or reject each parameter set. The posterior parameter distribution is based on the Bayesian formulation:

$$
f(\boldsymbol{p})=\eta L(\boldsymbol{p}) \rho(\boldsymbol{p})
$$

where $L(\boldsymbol{p})$ is the likelihood function, $\rho(\boldsymbol{p})$ is the prior probability density for the vector of parameters, and $\eta$ is a normalisation constant (refer Knorr and Kattge ${ }^{24}$, Mosegaard and Sambridge ${ }^{25}$, along with the Supplementary Information, for full details).

A set of 11 parameters was selected for the MCMH processing on the basis of the uncertainty analysis explained above. Prior parameter distributions were established based on 
previous studies and existing model calibrations ${ }^{6,9}$. Gaussian uncertainties were assumed for both prior parameters and the observations. Our method included boundary values that result in truncated normal distributions for the model parameters, with boundaries selected to restrict the parameter space to physically realistic settings. All of the data, except the ocean temperature change profile, were organised into decadal averages to smooth out natural variability, since MAGICC does not simulate this feature of the climate system. Measurement uncertainties were obtained from the respective data sets in addition to a component for the natural variability to derive an overall observational standard deviation; these formed the diagonal elements of the error covariance matrix.

We analysed the error residuals from an initial run and found that they were well represented by a correlation time-scale of 30 years. The MCMH algorithm was then re-run, using these correlations. It was executed 50,000 times to obtain 37,926 accepted parameter sets, where the relatively high acceptance rate reflects the quality of the estimated priors. The resulting posterior distribution can then be used in conjunction with a given emission scenario to produce probabilistic global-mean temperature change projections.

\section{References}

1. Nakicenovic, N. \& Swart, R. IPCC Special Report on Emissions Scenarios (Cambridge University Press, Cambridge, UK, 2000).

2. Meehl, G. et al. The WCRP CMIP3 multi-model dataset: A new era in climate change research. Bull. Am. Meteorol. Soc. 88, 1383-1394 (2007).

3. Friedlingstein, P. et al. Climate-carbon cycle feedback analysis: results from the C4MIP 
model intercomparison. Journal of Climate 19, 3337-3353 (2006).

4. Collins, M. et al. Climate model errors, feedbacks and forcings: a comparison of perturbed physics and multi-model ensembles. Climate Dynamics 36, 1737-1766 (2011).

5. Forest, C. E., Stone, P. H. \& Sokolov, A. P. Constraining climate model parameters from observed 20th century changes. Tellus 60A, 911-920 (2008).

6. Meinshausen, M. et al. Greenhouse-gas emission targets for limiting global warming to $2^{\circ}$ C. Nature 458, 1158-1162 (2009).

7. Ricciuto, D. M., Davis, K. J. \& Keller, K. A Bayesian calibration of a simple carbon cycle model: The role of observations in estimating and reducing uncertainty. Global Biogeochemical Cycles 22, GB2030 (2008).

8. Booth, B. B. B. et al. High sensitivity of future global warming to land carbon cycle processes. Environmental Research Letters 7 (2012).

9. Meinshausen, M., Raper, S. C. B. \& Wigley, T. M. L. Emulating coupled atmosphere-ocean and carbon cycle models with a simpler model, MAGICC6 - Part 1: Model description and calibration. Atmospheric Chemistry and Physics 11, 1417-1456 (2011).

10. Meinshausen, M. et al. The RCP greenhouse gas concentrations and their extensions from 1765 to 2300. Climatic Change 109, 213-241 (2011).

11. Rogelj, J., Meinshausen, M. \& Knutti, R. Global warming under old and new scenarios using IPCC climate sensitivity range estimates. Nature Climate Change 2, 248-253 (2012).

12. Bodman, R. W. Estimating uncertainties in future global warming using a simple climate model. Ph.D. thesis, The University of Melbourne (2011). 
13. Brohan, P., Kennedy, J. J., Harris, I., Tett, S. F. B. \& Jones, P. D. Uncertainty estimates in regional and global observed temperature changes: A new data set from 1850. Journal of Geophysical Research 111, D12106 (2006).

14. Domingues, C. M. et al. Improved estimates of upper-ocean warming and multi-decadal sea-level rise. Nature 453, 1090-1093 (2008).

15. Wijffels, S. E. Vertical ocean temperature change profile, 1960-2008 (personal communication). CSIRO Wealth from Oceans Flagship, Hobart, Tasmania, Australia (2010).

16. Masarie, K. A. \& Tans, P. P. Extension and integration of atmospheric carbon dioxide data into a globally consistent measurement record. Journal of Geophysical Research 100, 11,593-11,610 (1995). URL withupdatesathttp://www.esrl.noaa.gov/ gmd/ccgg/trends/global.html.

17. Tanaka, K. \& Raddatz, T. Correlation between climate sensitivity and aerosol forcing and its implication for the "climate trap". Climatic Change 109, 815-825 (2011).

18. Hansen, J. E. A slippery slope: How much global warming constitutes "dangerous anthropogenic interference”. Climatic Change 68, 269-279 (2005).

19. Schneider, S. H. \& Lane, J. An overview of 'dangerous' climate change. In Schellnhuber, H. J., Cramer, W., Nakicenovic, N., Wigley, T. M. L. \& Yohe, G. (eds.) Avoiding Dangerous Climate Change, chap. 2 (Cambridge University Press, 2006).

20. Knutti, R. et al. A review of uncertainties in global temperature projections over the twenty-first century. Journal of Climate 21, 2651-2662 (2008). 
21. Tarantola, A. Inverse Problem Theory and Methods for Model Parameter Estimation (Society for Industrial and Applied Mathematics, 2005).

22. Frame, D. J. et al. Constraining climate forecasts: the role of prior assumptions. Geophysical Research Letters 32, L09702 (2005).

23. Rayner, P. J., Koffi, E., Scholze, M., Kaminski, T. \& Dufresne, J.-L. Constraining predictions of the carbon cycle using data. Philosophical Transactions of the Royal Society 369, 1955-1966 (2011).

24. Knorr, W. \& Kattge, J. Inversion of terrestrial ecosystem model parameter values against eddy covariance measurements by Monte Carlo sampling. Global Change Biology 11, 1333-1351 (2005).

25. Mosegaard, K. \& Sambridge, M. Monte Carlo analysis of inverse problems. Inverse Problems 18, R29-R54 (2002).

Acknowledgments We gratefully thank Malte Meinshausen and Jens Kattge for supplying the MAGICC and MCMH code respectively. This research was supported by the Australian Research Council through the Discovery Projects funding scheme (project number FF0668679) and an ARC Professorial Fellowship (DP1096309).

Author contributions R.B, P.R. and D.K designed the research. R.B. carried out the analysis, with P.R. adding the linear uncertainty analysis of the posterior covariance. R.B. wrote the paper. All authors discussed the results and edited the manuscript.

Additional information Supplementary Information accompanies this paper on www . nature.com/ natureclimatechange. Reprints and permissions information is available online at www . nature. 
com/reprints. Correspondence and requests for materials should be addressed to R.B. (email:

roger.bodman@vu.edu.au). Results are archived at http://hdl.handle.net/102.100.100/ 9790.

286 Competing financial interests The authors declare that they have no competing financial interests. 
Table 1: Relative contributions to scientific uncertainty from MAGICC's primary climate-carbon cycle parameters towards global-mean temperature change in 2100 with A1FI emissions. Parameter groups: 4 climate system, 1 combined aerosol and 6 carbon cycle parameters that account for $96 \%$ of the uncertainty from a total of 29 selected parameters.

\begin{tabular}{lr} 
Parameter name & $\begin{array}{c}\text { uncertainty } \\
\text { contribution } \%\end{array}$ \\
\hline climate sensitivity $\Delta T_{2 \mathrm{x}}$ & $58.4 \%$ \\
ocean vertical diffusivity $K$ & $2.0 \%$ \\
land ocean warming ratio $R_{\mathrm{lo}}$ & $1.7 \%$ \\
land ocean heat coefficient $\kappa_{\mathrm{lo}}$ & $0.6 \%$ \\
\hline sulfate aerosol forcing in $2005 A F_{\mathrm{sox}}$ & $4.7 \%$ \\
\hline $\mathrm{CO}_{2}$ fertilisation factor $\beta_{s}$ & $8.4 \%$ \\
temperature feedback factor, respiration $\sigma_{\mathrm{R}}$ & $8.0 \%$ \\
temperature feedback factor, NPP $\sigma_{\mathrm{NPP}}$ & $7.4 \%$ \\
carbon flux partition, NPP to plant $g_{\mathrm{P}}$ & $2.5 \%$ \\
ocean cc impulse response scale factor $O_{\mathrm{r}}$ & $2.2 \%$ \\
carbon flux partition, plant to detritus $q_{\mathrm{H}}$ & $0.1 \%$ \\
\hline
\end{tabular}

Table 2: SRES A1FI CO ${ }_{2}$ concentrations and global-mean temperature change results at 2100 relative to 1980-1999 for the 'Combined', 'Without' ( i.e., without carbon cycle observations), and 'Hybrid' cases.

\begin{tabular}{rccc} 
& 'Combined' & 'Without' & 'Hybrid' \\
\hline & \multicolumn{3}{c}{$\mathrm{CO}_{2}$ concentration, ppm } \\
median & 943 & 1070 & 1037 \\
mode & 907 & 958 & 960 \\
likely range & $803-1140$ & $851-1408$ & $807-1399$ \\
& \multicolumn{4}{c}{ Temperature change, ${ }^{\circ} \mathrm{C}$ (relative to $\left.1980-1999\right)$} \\
median & 4.2 & 4.5 & 4.5 \\
mode & 4.1 & 4.1 & 4.4 \\
likely range & $2.9-5.7$ & $3.1-6.2$ & $3.0-6.5$ \\
\hline
\end{tabular}


Table 3: $\mathrm{CO}_{2}$ concentrations and global-mean temperature change in 2100 relative to 1980-1999 for three SRES emission scenarios, A1B, A1FI and A2, with results for the prior and posterior parameter distributions, with and without the carbon cycle temperature feedbacks (without temperature feedbacks corresponds to setting the four temperature feedback factors for respiration $\sigma_{\mathrm{R}}$, net primary productivity (NPP) $\sigma_{\mathrm{NPP}}$, detritus $\sigma_{\mathrm{Q}}$ and soil $\sigma_{\mathrm{U}}$, to zero.)

\begin{tabular}{|c|c|c|c|c|}
\hline & \multicolumn{2}{|c|}{ Prior dist'n } & \multicolumn{2}{|c|}{ Post. dist'n } \\
\hline $\begin{array}{l}\text { Carbon cycle temperature } \\
\text { feedbacks: }\end{array}$ & On & Off & On & Off \\
\hline \multicolumn{5}{|c|}{ A1B emission scenario } \\
\hline & \multicolumn{4}{|c|}{$\mathrm{CO}_{2}$ concentration, $\mathrm{ppm}$} \\
\hline median & 752 & 659 & 704 & 641 \\
\hline mode & 663 & 646 & 673 & 622 \\
\hline \multirow{2}{*}{ likely range } & $595-1050$ & $571-761$ & $612-846$ & $576-717$ \\
\hline & \multicolumn{4}{|c|}{ Temperature change, ${ }^{\circ} \mathrm{C}$ relative to $1980-1999$} \\
\hline median & 3.6 & 3.2 & 3.1 & 2.8 \\
\hline mode & 2.7 & 3.1 & 2.9 & 2.8 \\
\hline \multirow[t]{2}{*}{ likely range } & $1.9-5.8$ & $1.8-4.6$ & $2.1-4.4$ & $1.9-3.9$ \\
\hline & \multicolumn{4}{|c|}{ A1FI emission scenario } \\
\hline & \multicolumn{4}{|c|}{$\mathrm{CO}_{2}$ concentration, ppm } \\
\hline median & 1010 & 882 & 943 & 856 \\
\hline mode & 914 & 869 & 907 & 831 \\
\hline \multirow[t]{2}{*}{ likely range } & $781-1383$ & $752-1020$ & $803-1140$ & $760-962$ \\
\hline & \multicolumn{4}{|c|}{ Temperature change, ${ }^{\circ} \mathrm{C}$ relative to $1980-1999$} \\
\hline median & 4.7 & 4.2 & 4.2 & 3.9 \\
\hline mode & 4.2 & 4.0 & 4.1 & 3.9 \\
\hline \multirow[t]{2}{*}{ likely range } & $2.6-7.0$ & $2.5-5.9$ & $2.9-5.7$ & $2.7-5.2$ \\
\hline & \multicolumn{4}{|c|}{ A2 emission scenario } \\
\hline & \multicolumn{4}{|c|}{$\mathrm{CO}_{2}$ concentration, ppm } \\
\hline median & 844 & 779 & 811 & 759 \\
\hline mode & 786 & 768 & 792 & 738 \\
\hline \multirow[t]{2}{*}{ likely range } & $691-1062$ & $673-895$ & $716-928$ & $681-847$ \\
\hline & \multicolumn{4}{|c|}{ Temperature change, ${ }^{\circ} \mathrm{C}$ relative to $1980-1999$} \\
\hline median & 3.3 & 3.0 & 3.1 & 2.8 \\
\hline mode & 3.0 & 2.8 & 3.0 & 2.8 \\
\hline likely range & $1.9-4.8$ & $1.8-4.2$ & $2.2-4.0$ & $2.0-3.7$ \\
\hline
\end{tabular}


Figure 1 Time series of global-mean temperature change for selected SRES marker scenarios: (a) A1B, (b) A1FI and (c) A2, as anomalies with respect to 1990 (19801999 mean) based on the posterior parameter distributions. Black line: median, shaded regions $66 \%$ (dark), 95\% (medium) and 99\% (light) confidence intervals. The uncertainty ranges at the sides are the IPCC likely range and best estimate (grey column) for 2090-2099 and our corresponding results (blue column); the black bars are the respective best estimate and mode (refer Supplementary Information Table S3 for numerical details).

Figure 2 Probability of exceeding $2^{\circ} \mathrm{C}$ global-mean temperature change relative to pre-industrial for A1FI (black line), A1B (blue line) and A2 (pale blue line) emission scenarios: (a) global-mean and (b) land-surface temperature change. 
(a)

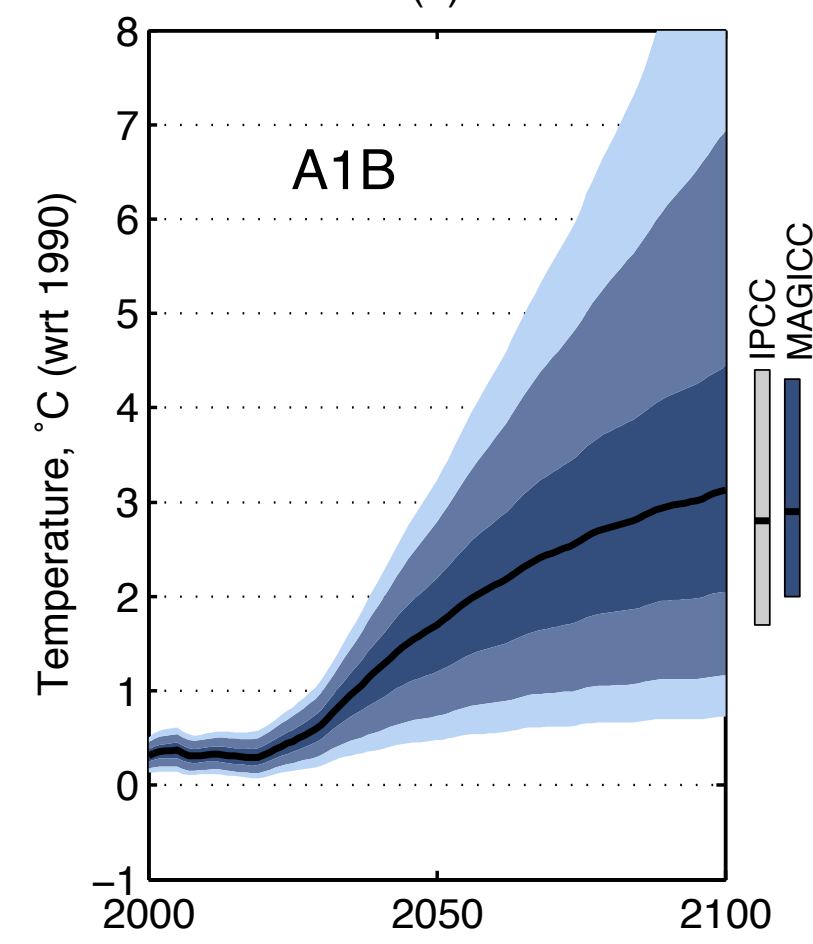

(b)

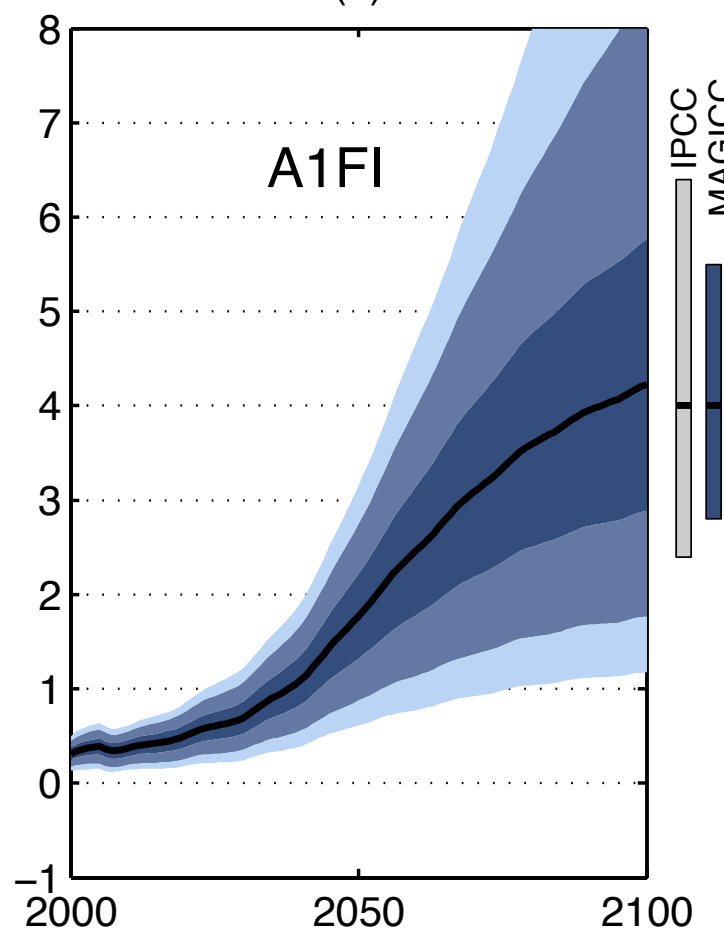

(c)

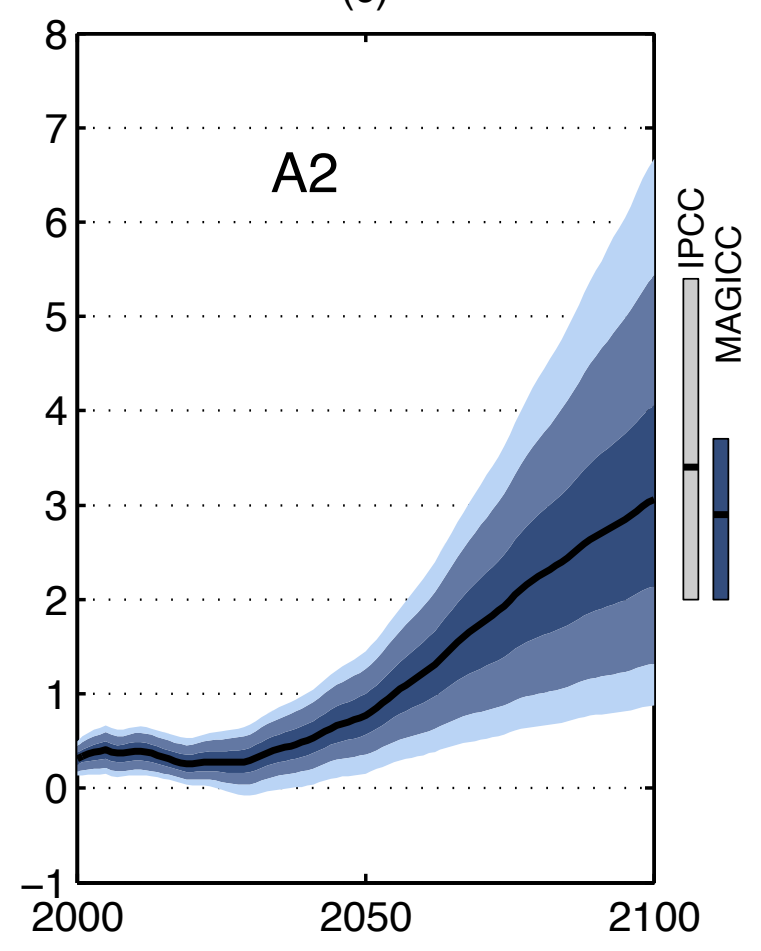


(a)

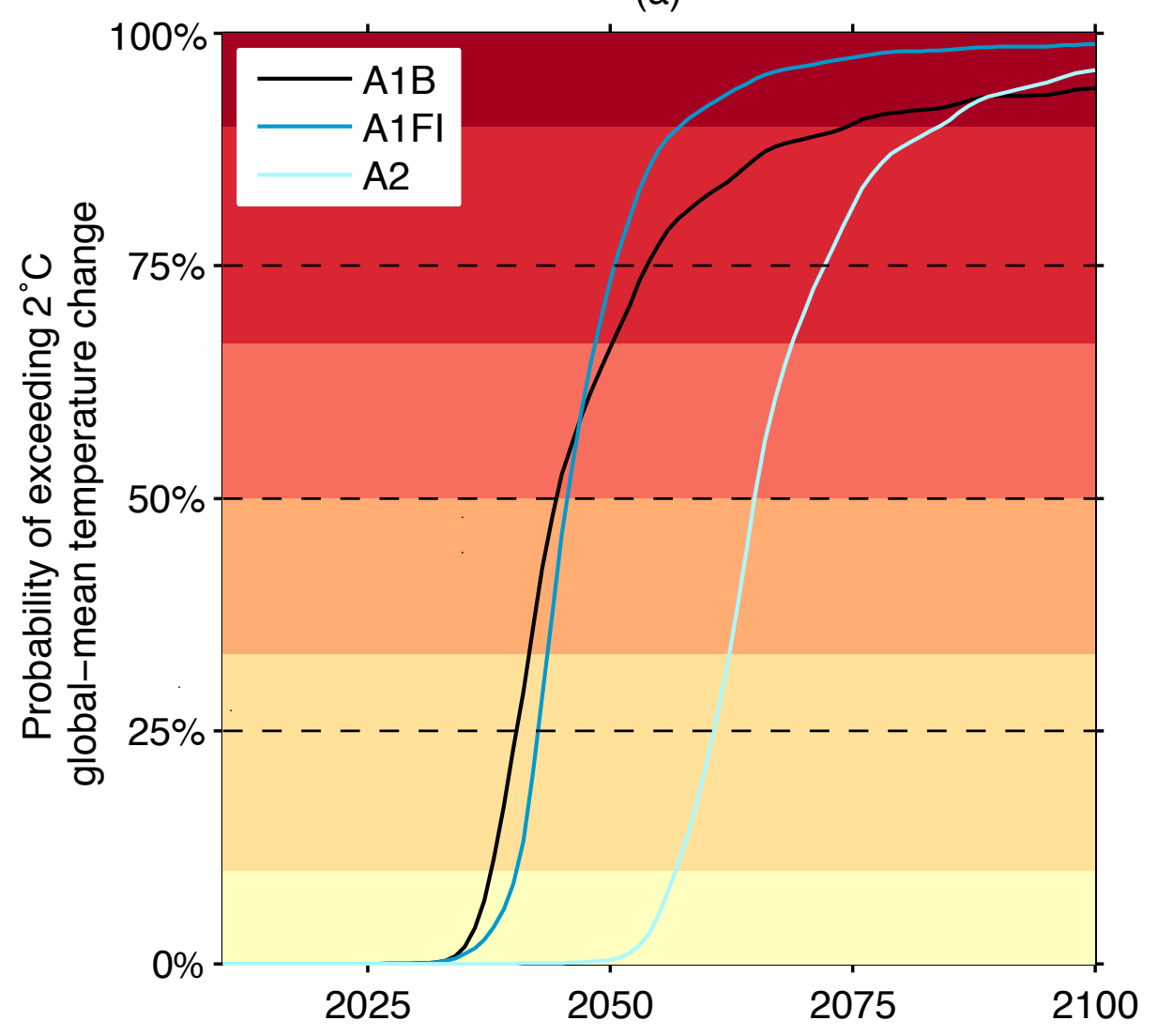

(b)

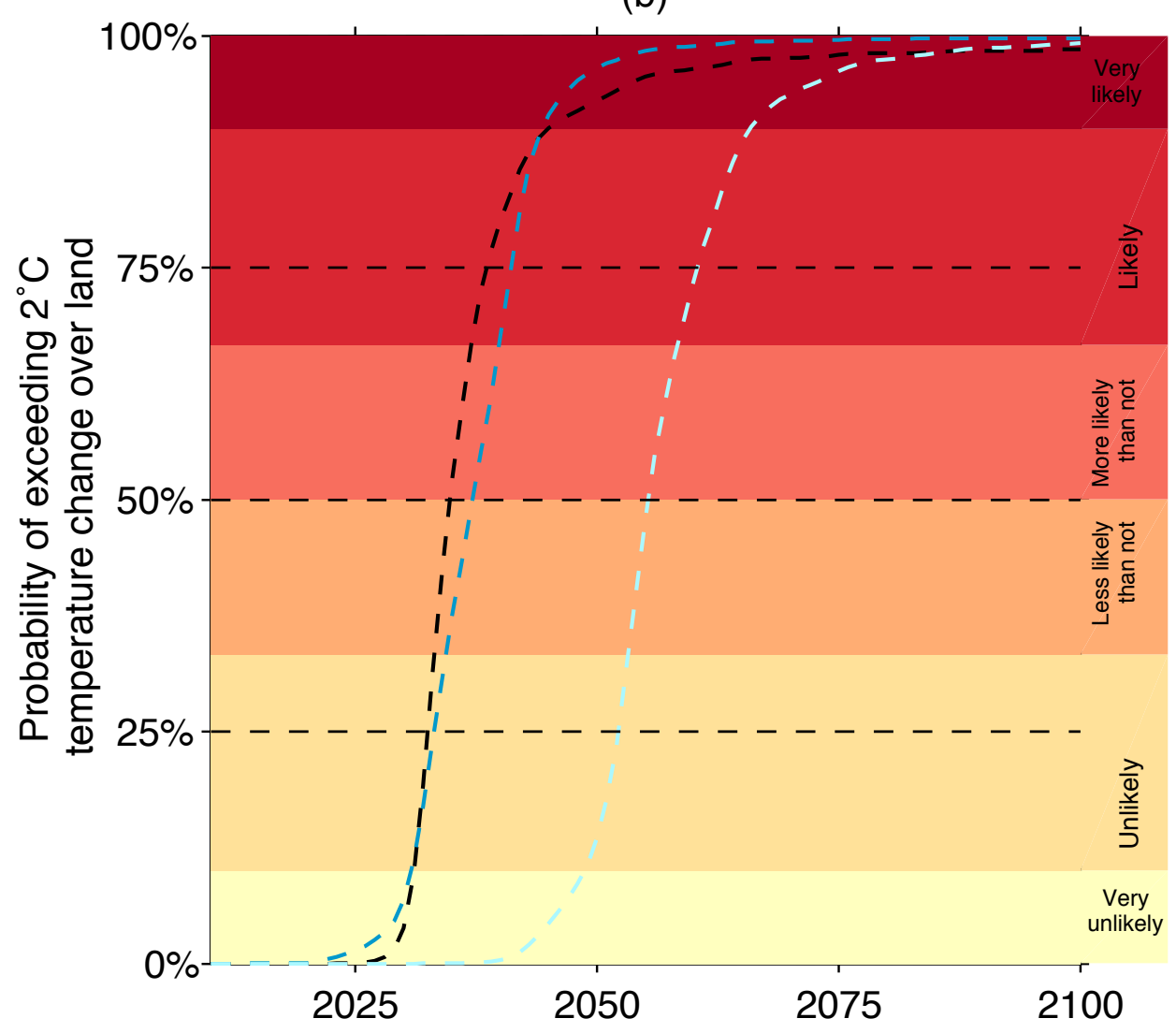

